\title{
Charge Transport in a Spin-Polarized 2D Electron System in Silicon
}

\author{
D. A. Knyazev, O. E. Omel'yanovskii, A. S. Dormidontov, and V. M. Pudalov \\ P. N. Lebedev Physical Institute, Leninski prospekt 53, Moscow, 119991 Russia
}

\begin{abstract}
The temperature dependences of the conductivity $\sigma(T)$ for strongly interacting $2 \mathrm{D}$ electron system in silicon have been analyzed both in zero magnetic field and in spin-polarizing magnetic field of $14.2 \mathrm{~T}$, parallel to the sample plane. Measurements were carried out in a wide temperature range $(1.4-9) \mathrm{K}$, in the ballistic regime of electron-electron interaction, i.e., for $T \tau>1$. In zero magnetic field, the data obtained for $\sigma(T)$ are quantitatively described by the theory of interaction corrections. In the fully spin-polarized state, the measured $\sigma(T)$ dependences are nonlinear and even nonmonotonic for the same temperature range, where the $\sigma(T)$ dependences are monotonic in the absence of the field. Nevertheless, the low-temperature parts of the experimental $\sigma(T)$ dependences are linear and are qualitatively consistent with the calculated interaction corrections.
\end{abstract}

PACS: 71.10.Ay, 75.47, 71.30.+h

Electron-electron interaction causes a number of significant effects in the conductivity and magnetoconductivity of $2 \mathrm{D}$ systems of charge carriers [1]: in particular (i) a strong metallic-like temperature dependence of conductivity $(d \sigma(T) / d T>0)$ in zero magnetic field [1-4], and (ii) strong magnetoresistance in the in-plain magnetic field [5-8].

At present, several models claim to explain these and other effects of the renormalization of the quasiparticle parameters by strong electron-electron interaction [2] both within the framework of the Fermi-liquid theory and beyond this framework. In this work, we compare with experimental data predictions (a) of the theory of interaction corrections [9] and, (b) of the two-phase model [10], where the $2 \mathrm{D}$ electron system is treated as consisting of a electron liquid with Wigner crystal inclusions. The former model qualitatively, and in some cases, even quantitatively reproduces the temperature dependence of the conductivity and magnetoconductivity of $2 \mathrm{D}$ systems [11-15] as a consequence of Fermi-liquid effects. The latter model qualitatively explains the above effects in conductivity from the essentially non Fermi-liquid viewpoint.

Investigation of the system in a magnetic field higher than the field of full spin polarization $B_{p o l}$, applied parallel to the $2 \mathrm{D}$ plane, can be a sensitive test for the above theories. This case is rather simple from the theoretical point of view (because parallel magnetic field affects only spins of electrons and does not affect their orbital motion), whereas the predictions of different theories for the spin-polarized state significantly differ from each other. For this reason, studies of electron transport in the completely spin polarized state can provide an additional key for understanding the properties of the strongly interacting $2 \mathrm{D}$ electron system.

Measurements of $\sigma(T)$ in the fully spin-polarized state have already been performed in Refs. $[16,17]$. In particular, it was reported in Ref. [16] that the conductivity in a magnetic field of $10 \mathrm{~T}$ is almost temperature independent in the temperature range $T=(0.3-3) \mathrm{K}$. This result seems to be in accord with the predictions of the two-phase model [10]. However, a field of $10 \mathrm{~T}$ was likely to be insufficiently strong for the complete spin polarization of the electron system in the studied density range $(1-2.5) \times 10^{11} \mathrm{~cm}^{2}$. The ratio $\sigma_{D}^{-1} d \sigma / d T$ of the derivatives measured in zero magnetic field and in the full spin polarization field was measured in Ref. [17] in a temperature range $(0.1-1.2) \mathrm{K}$. It was found that this ratio is consistent with the theory of temperature-dependent screening [18-21] and is inconsistent with calculated interaction corrections [9] for the ballistic regime. However, it is worth noting that the applied strong parallel field increases the effective disorder in the 2D system (reduces $\tau)$. For this reason, although the temperature range of measurements in zero magnetic field corresponded to the ballistic interaction regime $T \tau>1$ in both works [16,17], the same temperature range in the strong magnetic field corresponded to a transient region $T \tau \sim 1$ between the diffusive and ballistic interaction regimes. Thus, the comparison of the $\sigma(T)$ slopes in zero field and in strong field was performed for two different regimes of electronelectron interaction.

In this paper, we report measurements of the temperature dependence of conductivity performed for higher temperatures, $(1.4-9) \mathrm{K}$, and in a stronger magnetic field, $14.2 \mathrm{~T}$. Under such conditions, the measurements certainly corresponded to the ballistic interaction regime $T \tau>1$ both in zero magnetic field and in the field of the full spin polarization. The results of our $\sigma(T)$ measurements in strong magnetic field disagree with the non Fermi-liquid model [10] that predicts vanishing temperature dependence of conductivity in the spin-polarized state. Our data for zero magnetic field, as well as the data in Ref. [11], are quantitatively consistent with the theory of interaction corrections [9] with no free parameters. In the spin-polarized state, the temperature dependence of conductivity is only qualitatively consistent with calculated interaction corrections, whereas the slopes of the experimental $\sigma(T)$ curves differ from the theoretically 
predicted values by a factor of $1.2-2.8$.

Measurements were performed on a (100)-Si MOSFET structure with the inverse electron layer, $5 \times 0.8 \mathrm{~mm}^{2}$ rectangular channel, and maximum carrier mobility $\mu \approx$ $2 \mathrm{~m}^{2} / \mathrm{Vs}$ at $T=1.4 \mathrm{~K}$. The conductivity was measured by the standard four-terminal ac technique with a frequency $\approx 5 \mathrm{~Hz}$. The current passing through the sample was small enough $(I \approx 10 \mathrm{nA})$ to prevent the overheating of electrons. The sample plane was parallel to the magnetic field with an accuracy $\approx 1^{\prime}$. The orientation was controlled according to vanishing of the weak localization peak in $\rho_{x x}\left(B_{\perp}\right)$ when the sample plane rotated with respect to the magnetic field direction.

\section{A. Measurements of $\sigma(T)$ at zero magnetic field}

Prior to studying conductivity in strong magnetic fields, we have done $\sigma(T, B)$ measurements in zero magnetic field and compared the results with theory. Figure 1 shows the $\rho(T)$ dependences in zero magnetic field for various electron densities. In the absence of the field, the sample resistivity strongly depends on temperature (see Fig. 1), exhibiting metallic conductivity typical for high mobility samples for low carrier densities (i.e., for strong electron-electron interaction).

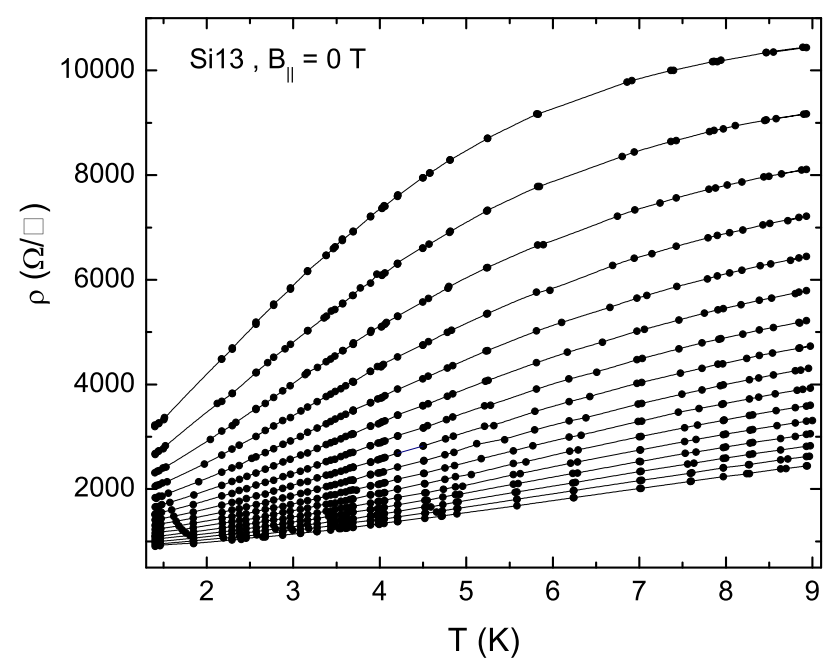

FIG. 1. Temperature dependence of $\rho(T)$ for densities 1.92, $2.04,2.16,2.28,2.39,2.51,2.63,2.75,2.86,2.98,3.10,3.22$, $3.34,3.45,3.57,3.69$ (from top to bottom, in $10^{11} \mathrm{~cm}^{-2}$ ). Magnetic field $B_{\|}=0 \mathrm{~T}$.

Figure 1 clearly shows linear regions in the $\rho(T)$ dependences at low temperatures. Low resistivity values indicate that the density range under investigation belongs to the metallic region $\left(\rho_{D} \ll h / e^{2}\right)$ and the conditions of the applicability of the interaction corrections theory [9] are satisfied. Figure 2 shows a comparison of the conductivity (recalculated from the low-temperature data shown in Fig. 1) with interaction corrections [9]:

$$
\sigma(T)=\sigma_{D}+\delta \sigma_{C}+15 \delta \sigma_{T},
$$

where $\sigma(T)$ is the Drude conductivity; $\delta \sigma_{C}$ and $\delta \sigma_{T}$ are the singlet and triplet terms of the interaction correction, respectively; and the factor 15 appears due to the twofold degeneracy in spin and valleys [22]. The correction associated with weak localization is small in this temperature range [23] and is disregarded in the calculation.

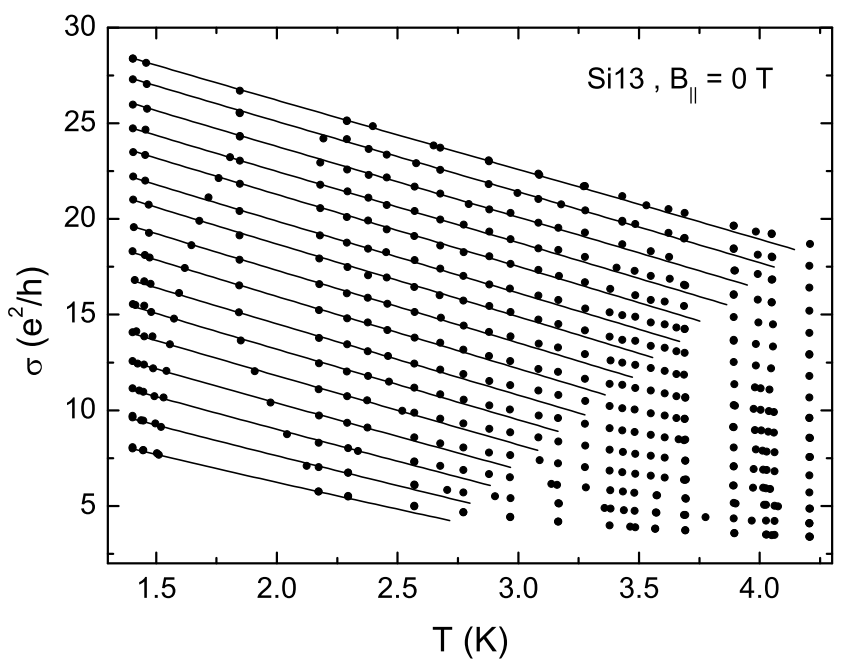

FIG. 2. Comparison of the experimental $\sigma(T)$ dependences (dots) and calculated interaction corrections Eq. (1) (solid lines) for densities 1.92, 2.04, 2.16, 2.28, 2.39, 2.51, 2.63, 2.75, $2.86,2.98,3.10,3.22,3.34,3.45,3.57,3.69$ (from bottom to top, in $\left.10^{11} \mathrm{~cm}^{-2}\right)$. Magnetic field $B_{\|}=0 \mathrm{~T}$.

The Fermi-liquid constant $F_{0}^{\sigma}$ entering into the triplet term $\delta \sigma_{T}$ was measured independently [24]. The Drude conductivity $\sigma_{D}$ was determined by extrapolating the experimental $\sigma(T)$ data to $T=0 \mathrm{~K}$ according to theoretical dependence (1) for the ballistic regime; $\tau$ was determined from $\sigma_{D}$ using the band mass $m^{*}=0.205 m_{e}$ (where $m_{e}$ is the electron mass) [24].

It is seen in Fig. 2 that, as well as in previous works [11-13], the calculated interaction corrections quantitatively describe the low-temperature regions of the experimental $\sigma(T)$ curves with no free parameters over the whole studied range of electron densities.

\section{B. Measurements of $\sigma(T)$ in a strong magnetic field}

In order to compare the transport properties of the system in the spin-polarized state and in zero magnetic field, we measured the $\rho(T)$ dependences in the in-plane 
magnetic field $B_{\|}=14.2 \mathrm{~T}$ for the same electron densities as in zero magnetic field. The experimental dependences of $\rho(T)$ for various electron densities in magnetic field $B_{\|}=14.2 \mathrm{~T}$ are shown in Fig. 3 .

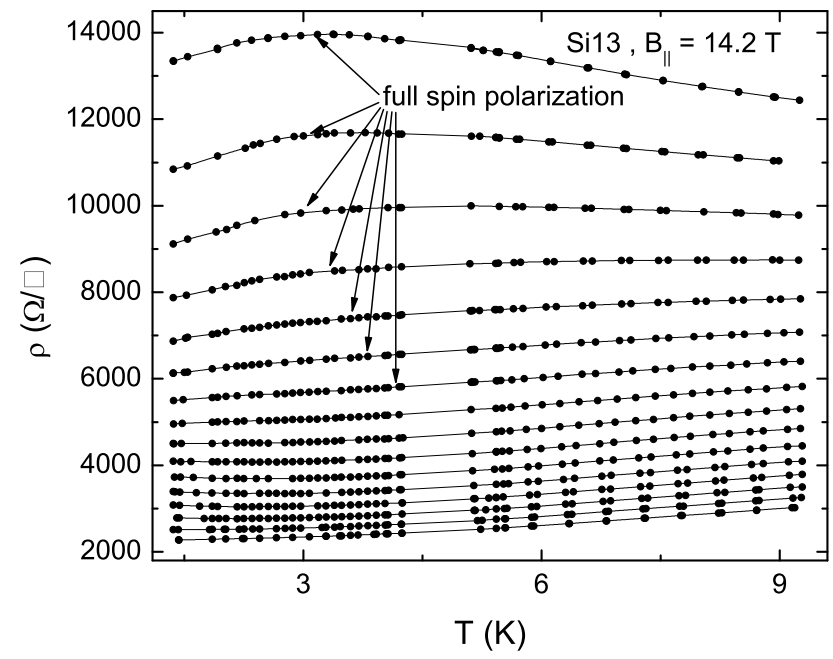

FIG. 3. Temperature dependences of $\rho(T)$ for carrier densities $1.92,2.04,2.16,2.28,2.39,2.51,2.63,2.75,2.86,2.98$, $3.10,3.22,3.34,3.45,3.57,3.69$ (from top to bottom, in $\left.10^{11} \mathrm{~cm}^{-2}\right)$. Magnetic field $B_{\|}=14.2 \mathrm{~T}$. The arrows mark the curves for the full spin polarization of the $2 \mathrm{D}$ system (for densities 1.92, 2.04, 2.16, 2.28, 2.39, 2.51, 2.63).

First, it is seen that the temperature dependences $\rho\left(T, B_{\|}=14.2 \mathrm{~T}\right)$ are substantially weaker than those for zero magnetic field (Fig. 1). It is also seen that the magnetic field increases the effective disorder in the system, which is manifested in the factor of four increase in resistivity with respect to the data shown in Fig. 1. This fourfold increase in resistivity agrees with the theory of temperature-dependent screening [20,21], which attributes it to an increase in $k_{F}$ by a factor of $\sqrt{2}$, as well as to a decrease in the density of electron states and to the factor of two increase in the screening radius.

Second, the increase in the temperature range of measurements enabled to reveal that the $\rho(T)$ dependences in a strong parallel magnetic field are nonmonotonic (see Fig. 3). Despite the $\rho(T)$ dependences, in general, are nonlinear (see Fig. 3), one can clearly see that for all densities at low-temperatures they have linear regions, which can be compared to the interaction corrections [9]. The $\rho(T)$ curves marked with arrows in Fig. 3 correspond to the condition of full spin polarization $g \mu_{B} B_{\|}>2 E_{F}$. The low-temperature regions of these curves recalculated to conductivity $\sigma(T)$ are shown in Fig. 4 in comparison with the interaction corrections calculated for the spinpolarized state $[25]$ :

$$
\sigma(T)=\sigma_{D}+\delta \sigma_{C}+3 \delta \sigma_{T}
$$

A decrease in the number of the triplet terms from $\left(2 n_{v}\right)^{2}-1=15$ to $n_{v}^{2}-1=3$ is caused by the removal of spin degeneracy at the unchanged twofold valley degeneracy $n_{v}=2[22]$. In the calculations of interaction corrections for the spin-polarized state, we used the same $F_{0}^{\sigma}$ and $m^{*}$ values as those for the case of zero magnetic field [26,27]. In view of an increase in disorder in the system for the spin-polarized state, we redetermined the $\tau$ and $\sigma_{D}$ values by extrapolating the $\sigma\left(T, B_{\|}=14.2 \mathrm{~T}\right)$ curves to $T=0 \mathrm{~K}$. We emphasize that, although $\tau$ values decreased, the temperature range of measurements remained to correspond to the ballistic interaction regime.

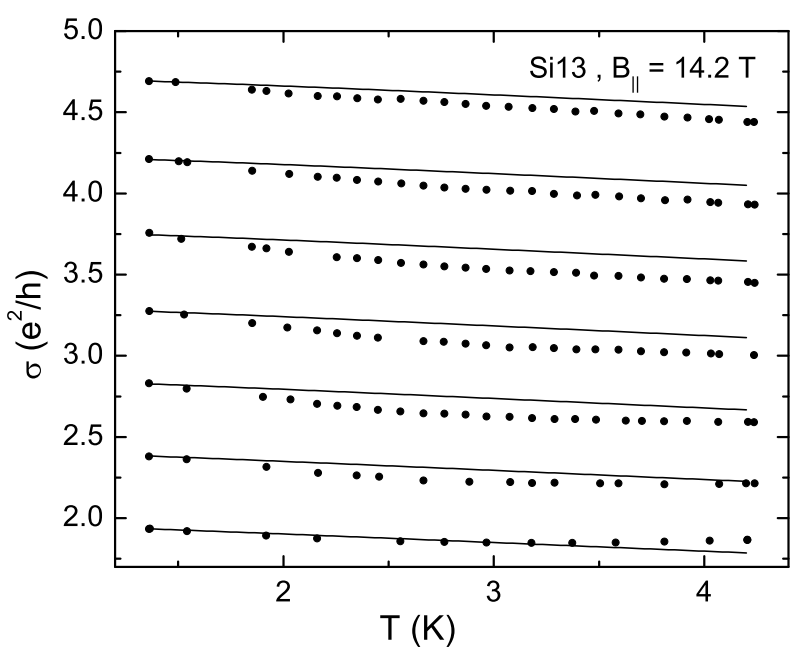

FIG. 4. Comparison of the experimental $\sigma(T)$ dependences (dots) and calculated interaction corrections (solid lines) (2) for the densities (from bottom to top) 1.92, 2.04, 2.16, 2.28, $2.39,2.51,2.63$ (in units of $10^{11} \mathrm{~cm}^{-2}$ ). Magnetic field $B_{\|}=14.2 \mathrm{~T}$.

It is seen from Fig. 4 that the experimental data qualitatively agree with interaction corrections. Although the temperature dependences $\sigma(T)$ in the spin-polarized state are noticeably (a factor of three to five) weaker than those for zero magnetic field (see Fig. 2), their slopes $d \sigma / d T$ are nonzero, which contradicts the predictions of the non Fermi-liquid two-phase model [10]. For the lowest densities (lower lines in Fig. 4), the discrepancy between the measured and theoretical slopes approximately amounts to $20-30 \%$. Such a discrepancy appears probably because the interaction corrections were calculated in Ref. [9] for the region $\sigma_{D} h / e^{2} \gg 1$, whereas $\sigma_{D} h / e^{2} \sim 2-3$ for the lowest curves in Fig. 4. For higher densities (upper lines in Fig. 4), the slopes of the experimental lines differ from the calculated values by a factor of $2-2.8$. The increase in the discrepancy with electron density is probably associated 
with a decrease in the spin polarization degree at finite temperature: $\left|g \mu_{B} B_{\|}-2 E_{F}\right| \sim T$. On the other hand, the theory of temperature-dependent screening $[19,20]$ predicts that $\left(d \sigma\left(T, B_{\text {pol }}\right) / d T\right) /(d \sigma(T, 0) / d T)=$ $1 / 2\left(\sigma_{D}\left(B_{p o l}\right) / \sigma_{D}(0)\right)=1 / 8$; however, according to the experimental data (see Figs. 2,4), this ratio varies only slightly for various densities and is equal to $\approx 0.04$, i.e., approximately one third of the predicted value.

Thus, in this work we measured the temperature dependence of the conductivity of the $2 \mathrm{D}$ electron system in $\mathrm{Si}$, both in the absence of a magnetic field and in the spin-polarized state. It has been found that the temperature dependence of conductivity in the spin-polarized state is nonmonotonic, which is not explained by any available theories. For low temperatures (but in the ballistic interaction regime, $T \tau>1$ ), the temperature dependence of conductivity is approximately linear both in zero magnetic field and in the field of full spin polarization; this enables one to compare the experimental data with theoretical predictions. The temperature dependence of the conductivity in the spin-polarized state is much weaker than that for the case of zero magnetic field, but it does not vanish. This result contradicts the two-phase model [10]. The $\sigma(T)$ dependences measured in zero magnetic field are quantitatively described by the theory of interaction corrections [9], whereas in the spin polarized state the agreement with theory is only qualitative. For both cases, the comparison with the theory is performed with no free parameters. The measured ratio $\left(d \sigma\left(T, B_{\text {pol }}\right) / d T\right) /(d \sigma(T, 0) / d T)$ of the slopes of the temperature dependences in the spin-polarizing and zero magnetic fields is larger than the values predicted by the interaction corrections theory by a factor of $\approx 1.2-3$ and is about one third of the value predicted by the theory of temperature-dependent screening.

The work was supported by the grants from RFBR, INTAS, Russian Academy of Sciences, the Presidential Program for the support of Young Russian researchers and Leading scientific schools. D.A.K. acknowledges the support of the Educational-Scientific Center, Lebedev Physical Institute, and Russian Science Support Foundation.

[1] E. Abrahams, S. V. Kravchenko, and M. P. Sarachik, Rev. Mod. Rhys. 73, 251 (2001); V. M. Pudalov, in: The Electron Liquid Paradigm in Condensed Matter Physics ed. by G. F. Giuliani and G. Vignale (IOS press, Amsterdam, 2004).

[2] V.M. Pudalov, M. E. Gershenson, H. Kojima, Chapter 19 in: Fundamental problems of mesoscopic physics, NATO science series, Ed. by I. Lerner, B. Altshuler, and Y.Gefen, p. 309 (Kluwer Academic Publ., Dordrecht 2004).
[3] S. V. Kravchenko, G. V. Kravchenko, J. E. Furneaux, V. M. Pudalov, M. D'Iorio, Phys. Rev. B 50, 8039 (1994).

[4] S. V. Kravchenko, W. E. Mason, G. E. Bowker, J. E. Furneaux, V. M. Pudalov, M. D'Iorio, Phys. Rev. B 51, 7038 (1995).

[5] D. Simonian, S. V. Kravchenko, M. P. Sarachik, V. M. Pudalov, Phys. Rev. Lett. 79, 2304 (1997).

[6] V. M. Pudalov, G. Brunthaler, A. Prinz, G. Bauer, 65, 932 (1997).

[7] V. M. Pudalov, G. Brunthaler, A. Prinz, G. Bauer, Physica B 249-251, 697 (1998).

[8] T. Okamoto, K. Hosoya, S. Kawaji, A. Yagi, Phys. Rev. Lett. 82, 3875 (1999).

[9] G. Zala, B. N. Narozhny, I. L. Aleiner, Phys. Rev. B 64, 214204 (2001).

[10] B. Spivak, Phys. Rev. B 64, 085317 (2001).

[11] V. M. Pudalov, M. E. Gershenson, H. Kojima, G. Brunthaler, A. Prinz, G. Bauer, Phys. Rev. Lett. 91, 126403 (2003).

[12] Y. Y. Proskuryakov, A. K. Savchenko, S. S. Safonov, M. Pepper, M. Y.Simmons, D. A. Ritchie, Phys. Rev. B 68, 076406 (2002).

[13] A. A. Shashkin, S. V. Kravchenko, V. T. Dolgopolov, T. M. Klapwijk, Phys. Rev. B 66, 073303 (2002).

[14] S. A. Vitkalov, K. James, B. N. Narozhny, M. P. Sarachik, T. M. Klapwijk, Phys. Rev. B 67, 113310 (2003).

[15] E. B. Olshanetsky, V. Renard, Z. D. Kvon, J. C. Portal, N. J. Woods, J. Zhang, J. J. Harris, Phys. Rev. Lett. 89, 126403 (2002).

[16] Y. Tsui, S. A. Vitkalov, M. P. Sarachik, T. M. Klapwijk, Phys. Rev. B 71, 113308 (2005).

[17] A. A. Shashkin, E. V. Deviatov, V.T. Dolgopolov, A. A. Kapustin, S. Anissimova, A. Venkatesan, S. V. Kravchenko, T. M. Klapwijk, Phys. Rev. B 73, 115420 (2006).

[18] F. Stern, Phys. Rev. Lett. 44, 1469 (1980).

[19] A. Gold, V. T. Dolgopolov, Phys. Rev. B 33, 1076 (1986).

[20] V. T. Dolgopolov, A. Gold, 71, 42 (2000).

[21] S. Das Sarma, E. H. Hwang, Phys. Rev. B 72, 205303 (2005).

[22] The intervalley splitting is $\Delta_{v} \ll T$ over the entire temperature range, and therefore does not affect the number of triplet terms in Eqs. (1) and (2).

[23] G. Brunthaler, A. Prinz, G. Bauer, V. M. Pudalov, Phys. Rev. Lett. 87, 096802 (2001).

[24] V. M. Pudalov, M. E. Gershenson, H. Kojima, N. Butch, E. M. Dizhur, G. Brunthaler, A. Prinz, G. Bauer, Phys. Rev. Lett. 88, 196404 (2002).

[25] I. L. Aleiner, private communication.

[26] In Refs. [11,27] it was shown that the effective mass SiMOS structures is independent of the parallel magnetic field. The $F_{0}^{\sigma}\left(B_{\|}\right)$dependence on $B_{\|}$is manifested only in low magnetic fields; in the field of full spin polarization $F_{0}^{\sigma}$ is extrapolated to the same value as in zero magnetic field [2].

[27] A. A. Shashkin, M. Rahimi, S. Anissimova, S. V. Kravchenko, V.T. Dolgopolov, T. M. Klapwijk, Phys. Rev. Lett. 91, 046403 (2003). 